\title{
Design and Implementation of Vehicle Bluetooth system based on Embedded System
}

\author{
Qiu Bo, Lv Changwu \\ Jiangxi Environmental Engineering Vocational College,Ganzhou 341000,China
}

Key words: embedded system; Vehicle Bluetooth; BlueCode; communication protocol; operating system

\begin{abstract}
Bluetooth module has the characteristics of simple design, small size, better maintainability and so on, if it is used in vehicle equipment, it can achieve a lot of functions, which has important reference value for the research and development of driving safety equipment. Based on this a new Bluetooth system is proposed in this paper, taking embedded platform as integrated mode, using BlueCode as the communication protocol, and taking the WinCE as operating system, the effective connection of control terminal and vehicle equipment has been achieved in the system. The mobile terminal and the navigation system of the central control room touch screen are connected together by Bluetooth adapter in Bluetooth system. Mobile phone operation can be achieved through the touch screen, but also to achieve some of the operation through the intelligent voice control, such as locking the door, driving lights, etc., which effectively improves the safety of driving
\end{abstract}

\section{Introduction}

Traffic deaths have been one of the major causes of accidental deaths worldwide since the last century, more and more people pay attention to traffic safety. Simulation tests in the United States show that, making a phone call during driving causes the driver's attention to drop 20\%, if the conversation is important, down as much as $\$ 37 \%$, if the driver is not focused on driving, the risk is 4 times the normal driving risk. In order to avoid driving distraction caused by operating other devices, Bluetooth system is introduced into the vehicle equipment. Bluetooth can realize the vehicle equipment and control of a large screen, and single-chip communication can be used, the use of large screen or voice way on a lot of switch operation in the car, which is very important for the study of safety equipment for vehicle.

\section{The design of embedded Bluetooth system}

Due to the adoption of frequency hopping and fast recognition technology, Bluetooth has the characteristics of fast transmission and stable communication, so as to prevent the premature fading of the signal and ensure the stability of the communication link. Bluetooth uses frequency hopping in 79 frequency hopping channels within one second. The interference of frequency hopping is reduced to the minimum, and the interference of random noise is avoided in the process of communication.

Taking Bluetooth as a vehicle equipment, on the one hand a large screen can be used on the phone and other mobile devices, on the other hand voice control a lot of switch also can be achieved, the main design of the Bluetooth system, the overall design framework is shown in Fig.1. 


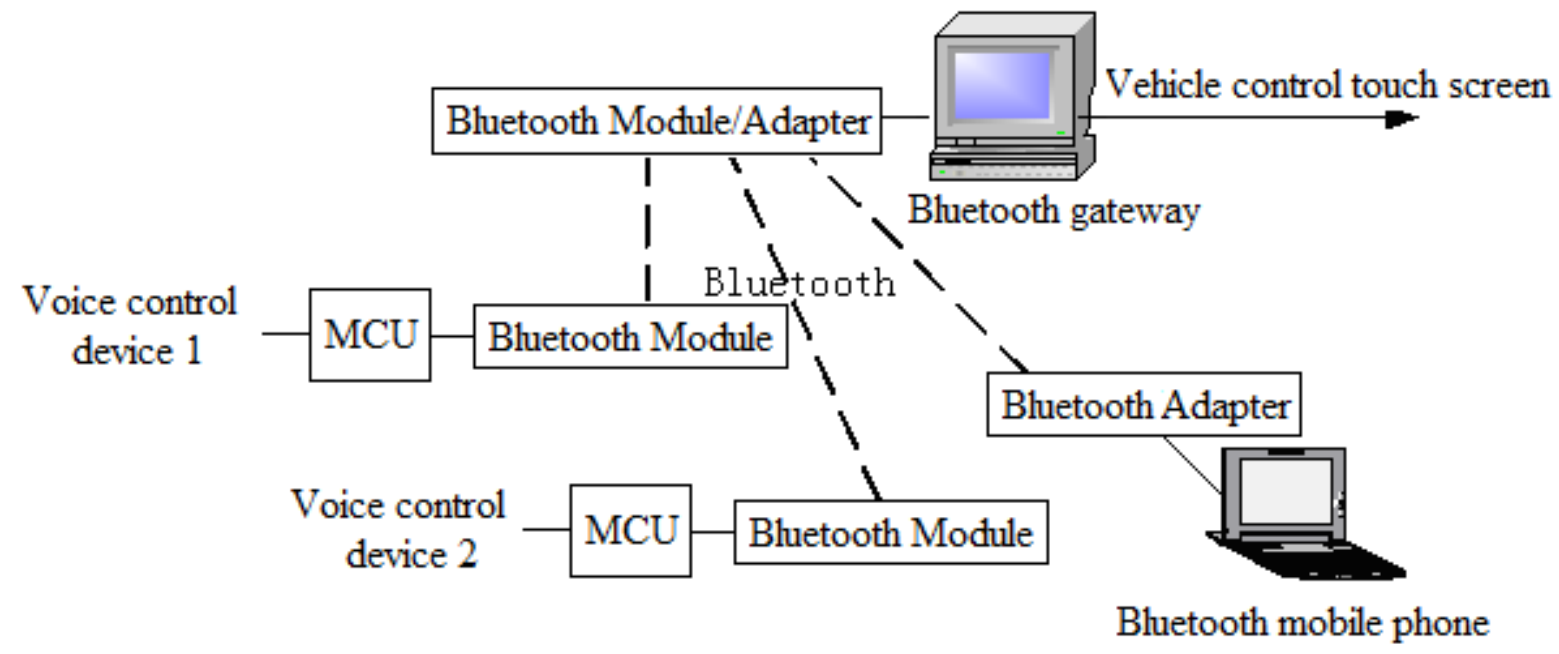

Fig.1 The HomeNet composition scheme based on Bluetooth Technology

As shown in Figure 1, Bluetooth adapter is connected with the large touch screen of the central console through one end of the Bluetooth grid, and the Bluetooth control module is connected with the Bluetooth mobile phone, Bluetooth control module can issue commands to the microcontroller, the car and the switching equipment. During the running time, can use the console touch screen devices or voice operated equipment for mobile phone and car, avoids the small screen operation or manual operation of distractions, and make the operation simple and easy.

\section{Application mode and program structure Bluetooth embedded vehicle system}

The design of Bluetooth embedded system with BlueCode protocol, the protocol supports HCI standard interface, users can be embedded in the Bluetooth system by using API function in a simple development environment integrated Bluetooth protocol.

Multiple applications can call the BlueCode function of API at the same time, so a parallel Bluetooth link can be built, which is shown in Figure 2.

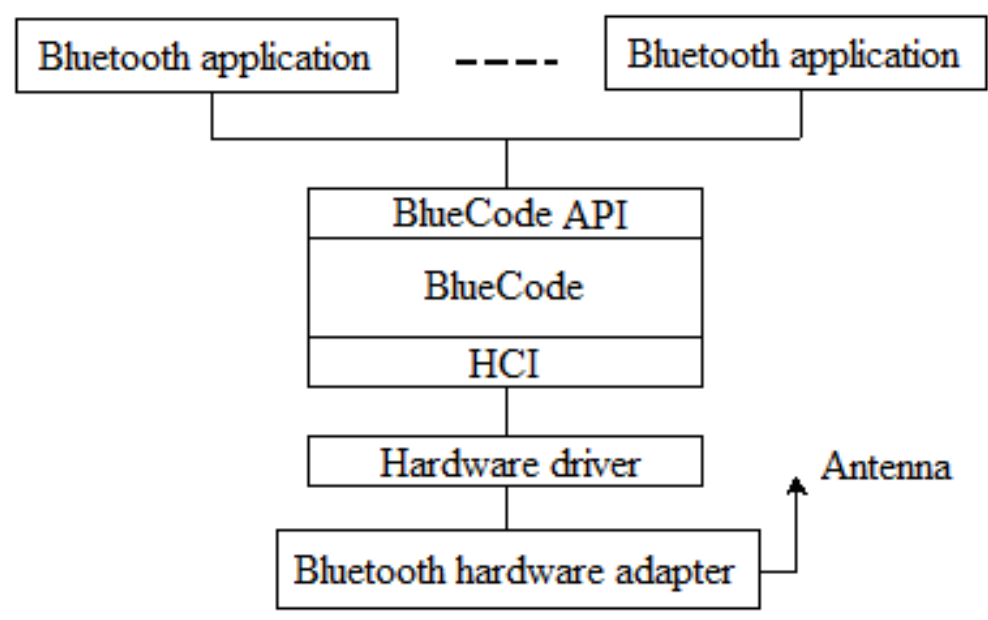

Figure 2 BlueCode application framework

The message and data transfer between the hardware module and the software module interface can only be carried out through the Bluetooth host controller interface, which is explained by HCI. The HCI layer is the interface between the hardware and software of the Bluetooth protocol, the protocol software above HCI layer protocol software is running on the host, and the function under HCI is completed by Bluetooth device, the two can interact each other through a transparent layer on both ends. 


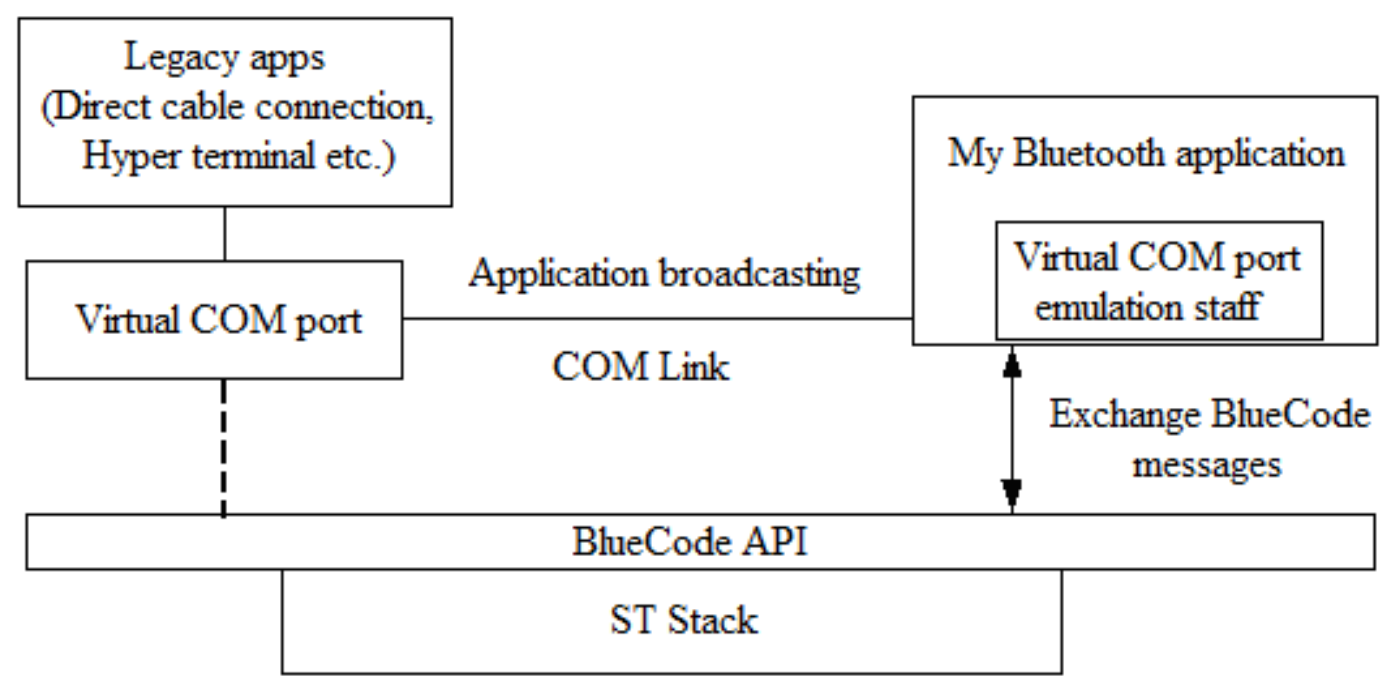

Fig.3 Bluetooth program structure

As shown in Figure 3, the sending thread and the receiving thread should be mainly considered in the development and design of the Bluetooth program structure, the sending thread is mainly responsible for receiving the data, and the data packet can be sent. The data receiving is to receive the data set into the cache when the Bluetooth system establishes the data connection, when the user receives the information queue, it will find the cached information, the user will receive the information and send the information to the specified function model.

\section{Embedded Bluetooth system communication test}

In order to verify the feasibility and reliability of embedded Bluetooth communication system, the performance of Bluetooth communication system was tested. The test is the main interference on the communication process of the interference signals, the signal receiver detection error rate, through the test, the test results are shown in Fig.4.

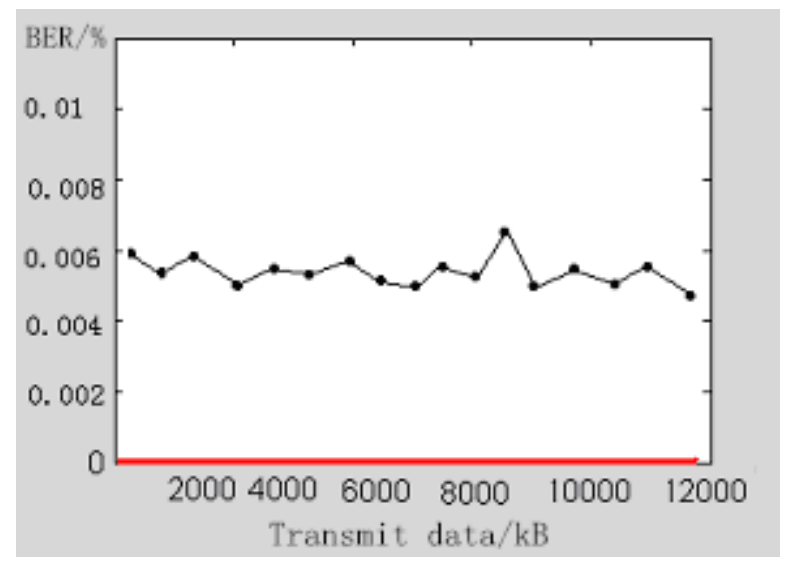

Fig.4 The comparison error rate of the system

In order to ensure patency of the Bluetooth communication, and avoid the influence of interference, multi hop Bluetooth technology has been used in the paper. When the system communication is disturbed, the bit error rate of the communication system of the Bluetooth system is analyzed. As shown in Figure 4, through statistical analysis, we found that the error rate of communication of Bluetooth system is greatly reduced by using multi hop technology, the stability of the communication and the fluency of the communication between the touch control screen and the voice control and vehicle equipment has been realized.

multi hop technology, Bluetooth communication system bit error rate is reduced greatly, ensure the 
stability of the communication, can screen and voice communication equipment and vehicle control to achieve fluency in touch.

\section{Conclusion}

In order to improve the traffic safety, a new Bluetooth system is designed, Bluetooth function of vehicle system has been realized, which is taking embedded platform as integrated mode, and taking the WinCE as operating system. The Bluetooth module designed in this paper has the characteristics of simple design, small size, strong maintainability. Through the communication ability test, it is found that the bit error rate of Bluetooth system is greatly reduced by using multi hop Technology. Therefore, it can guarantee the communication stability of the console touch screen and the user terminals such as voice control devices and mobile phones. Bluetooth enabled mobile phones, lights, doors and other operations become easier to operate, it can be tested in practical application to meet the market demand.

\section{Reference}

[1] Li Manling. Design of digital intelligent access control system based on NFC [J]. Journal of Ezhou University, Vol.21(6), (2014), p.108-110

[2] Chang Guoquan, Zhai Yan. Design of intelligent door lock control system based on Bluetooth [J]. Electronic Engineering \& Product World, No.05, (2015) , p.41-44

[3] Miao Chao, Chen Keming, Lu Jianguo, et al. Intelligent remote control door lock with wireless charging [J]. Journal of Hangzhou Dianzi University, Vol. 35(4), (2015), p.20-22

[4] Gao Jianhua, Hu Zhenyu. Application of Internet of things technology in intelligent building [J]. Architecture Technology, Vol.44 (2), (2013), p.136-137

[5] Sun Yongdao, Wang Yong, Zhang Lan. Design and implementation of a mobile phone access control system based on encrypted two-dimensional code [J]. Bulletin of Science and Technology, Vol.30(7), (2014), p.166-169

[6] Ma Xiang. Knowledge development in Web based on Java language [J]. Information Technology and Informatization, Vol.13 (15), (2015), p.169-171.

[7] Su Xianglin, Chen Wenyi, Yan sprinkle. The open platform of Internet of things based on raspberry pie [J]. Electronic Science and Technology, Vol.28 (9), (2015), p.35-37.

[8] Cui Yang, Zhang Weihua. An intelligent home control system based on Arduino[J]. Application of Electronic Technique, Vol.40 (4), (2014), p.123-125.

[9] Xue Liang, Zhang Jifei. Design of intelligent home security system based on IOS mobile terminal [J]. Computing Technology and Automation, Vol.35(2), (2016), p.89-92.

[10] Shen Weiguang, Jiang Jianfei, He Wei Feng. Development status and future trend of the high performance DSP [J]. China Integrated Circuit, Vol.413(4), (2011), p.20-25.

[11] Ding Youyuan, Wang Anmin. DSP software and hardware design based on multi core parallel processing [J]. Microcontroller \& Embedded Systems, Vol.158 (8), (2012) , p.43-45.

[12] Gao Feng, Bi Jingping, Guo Jingfeng, et al. The Android client of the vehicle state perception system[J]. Journal of Tsinghua University(Science and Technology), Vol.S1, (2011), p.1393-1396. 
[13] Zhang Yang, Liu Xinhui. Stability of articulated vehicle yaw stability [J]. Journal of Jilin University (Engineering and Technology Edition), No.02, (2012), p.266-271. 